\title{
Natural Wolbachia infection in field-collected Anopheles and other mosquito species from Malaysia
}

Meng Li Wong', Jonathan Wee Kent Liew', Wai Kit Wong², Sandthya Pramasivan', Norzihan Mohamed Hassan³, Wan Yusoff Wan Sulaiman' ${ }^{1}$, Nantha Kumar Jeyaprakasam', Cherng Shii Leong ${ }^{1}$, Van Lun Low ${ }^{4}$ and Indra Vythilingam ${ }^{1^{*}}$ (ID

\begin{abstract}
Background: The endosymbiont bacterium Wolbachia is maternally inherited and naturally infects some filarial nematodes and a diverse range of arthropods, including mosquito vectors responsible for disease transmission in humans. Previously, it has been found infecting most mosquito species but absent in Anopheles and Aedes aegypti. However, recently these two mosquito species were found to be naturally infected with Wolbachia. We report here the extent of Wolbachia infections in field-collected mosquitoes from Malaysia based on PCR amplification of the Wolbachia wsp and 165 rRNA genes.
\end{abstract}

Methods: The prevalence of Wolbachia in Culicinae mosquitoes was assessed via PCR with wsp primers. For some of the mosquitoes, in which the wsp primers failed to amplify a product, Wolbachia screening was performed using nested PCR targeting the 16S rRNA gene. Wolbachia sequences were aligned using Geneious 9.1.6 software, analyzed with BLAST, and the most similar sequences were downloaded. Phylogenetic analyses were carried out with MEGA 7.0 software. Graphs were drawn with GraphPad Prism 8.0 software.

Results: A total of 217 adult mosquitoes representing 26 mosquito species were screened. Of these, infections with Wolbachia were detected in 4 and 15 mosquito species using wsp and 165 rRNA primers, respectively. To our knowledge, this is the first time Wolbachia was detected using 165 rRNA gene amplification, in some Anopheles species (some infected with Plasmodium), Culex sinensis, Culex vishnui, Culex pseudovishnui, Mansonia bonneae and Mansonia annulifera. Phylogenetic analysis based on wsp revealed Wolbachia from most of the mosquitoes belonged to Wolbachia Supergroup B. Based on 165 rRNA phylogenetic analysis, the Wolbachia strain from Anopheles mosquitoes were more closely related to Wolbachia infecting Anopheles from Africa than from Myanmar.

Conclusions: Wolbachia was found infecting Anopheles and other important disease vectors such as Mansonia. Since Wolbachia can affect its host by reducing the life span and provide resistance to pathogen infection, several studies have suggested it as a potential innovative tool for vector/vector-borne disease control. Therefore, it is important to carry out further studies on natural Wolbachia infection in vector mosquitoes' populations as well as their long-term effects in new hosts and pathogen suppression.

Keywords: Wolbachia, Mosquitoes, wsp gene, 165 rRNA, Anopheles, Vectors

*Correspondence: indrav@um.edu.my

1 Department of Parasitology, Faculty of Medicine, University of Malaya, 50603 Kuala Lumpur, Malaysia

Full list of author information is available at the end of the article

(c) The Author(s) 2020. This article is licensed under a Creative Commons Attribution 4.0 International License, which permits use, sharing, adaptation, distribution and reproduction in any medium or format, as long as you give appropriate credit to the original author(s) and the source, provide a link to the Creative Commons licence, and indicate if changes were made. The images or other third party material in this article are included in the article's Creative Commons licence, unless indicated otherwise in a credit line to the material. If material is not included in the article's Creative Commons licence and your intended use is not permitted by statutory regulation or exceeds the permitted use, you will need to obtain permission directly from the copyright holder. To view a copy of this licence, visit http://creativeco mmons.org/licenses/by/4.0/. The Creative Commons Public Domain Dedication waiver (http://creativecommons.org/publicdomain/ zero/1.0/) applies to the data made available in this article, unless otherwise stated in a credit line to the data. 


\section{Background}

The endosymbiotic bacterium Wolbachia is maternally inherited and naturally infects some filarial nematodes and a diverse range of arthropods, including mosquito vector species which are responsible for disease transmission to humans [1, 2]. Wolbachia is found naturally in various mosquitoes, including Aedes, Culex, Armigeres, Mansonia, Coquillettidia, Culiseta, Hodgesia, Ochlerotatus, Tripteroides and Uranotaenia [3-8] but was believed to be absent in Aedes aegypti and Anopheles mosquitoes [3]. To enhance their spread across the population, Wolbachia has a wide host range as well as wide tissue distributions [9]. Besides, Wolbachia can possibly be transferred horizontally between mosquito populations though the mechanism involved is not fully understood [10].

Wolbachia infection in the host causes a broad range of abnormal reproductive phenotypes which include malekilling, parthenogenesis, feminization and cytoplasmic incompatibility (CI) $[11,12]$. Cytoplasmic incompatibility caused by Wolbachia infection is the most well-known and common phenotypic effect and is considered a potential vector control alternative since crosses between individuals with different Wolbachia infection status will result in few or no offspring [13]. This effect confers indirect reproductive advantages to the infected females as infected females can produce viable progenies, but uninfected females when mated with infected males produce fewer viable progenies, if any [14]. Therefore, CI, along with maternal transmission of Wolbachia, allows Wolbachia to spread rapidly through a population [14, 15]. Several factors affect the CI in the host, namely strain of Wolbachia, host species, temperature and rearing density [16-19].

Furthermore, some studies have shown that Wolbachia can inhibit pathogen replication within mosquitoes [20, 21] such as dengue virus (DENV) [22-28], chikungunya virus (CHIKV) [25, 29, 30], yellow fever virus (YFV) [30], Zika virus (ZIKV) [31-33], Plasmodium parasites [25, 34] and filarial nematodes [35]. In studying the impact of Wolbachia infection on Plasmodium infection in Anopheles, transiently and somatically infected Anopheles gambiae with wMelPop or wAlbB Wolbachia strains was found to have significant reductions in Plasmodium berghei or Plasmodium falciparum oocyst infections [36, 37]. Following the increasing findings of natural Wolbachia infections in Anopheles [38], it was also found that natural populations of Wolbachia-infected An. coluzzii females have a lower frequency of Plasmodium infections than Wolbachia-negative individuals [39]. Gomes et al. [40] further showed that naturally infected field $A n$. gambiae, has significantly lower Plasmodium sporozoite infections. These findings support the potential use of Wolbachia in controlling vector-borne disease transmission. Currently, in Malaysia, studies on the release of Wolbachia-infected Ae. aegypti are ongoing to curb the spread of dengue [41].

It was believed that Wolbachia is absent in Ae. aegypti and Anopheles mosquitoes [3] until the year 2014, when the $w$ Anga Wolbachia strain was detected in An. gambiae collected from West Africa [38]. This was followed by several other discoveries of Wolbachia in other Anopheles vectors in Africa [40, 42-45] and Southeast Asia (Myanmar) [46]. Moreover, recent studies have also found Wolbachia naturally infecting Ae. aegypti in the Philippines [47], Thailand [48], Malaysia [49], India [50], Florida [51], New Mexico [52], Texas [53] and Panama [54].

Amplification of Wolbachia genes such as Wolbachia surface protein (wsp), filamenting temperature-sensitive mutant $\mathrm{Z}$ (ftsZ) and $16 \mathrm{~S}$ rDNA using polymerase chain reaction (PCR) has been used to detect Wolbachia in the vector. Previous failure in detecting Wolbachia in Anopheles mosquitoes may be due to limitations in the detection system used such as non-optimal DNA amplification [38]. This has led to the development of a nested PCR amplification of $16 S$ rDNA region for Wolbachia detection in Anopheles mosquitoes [39]. A study by Marcon et al. [55] demonstrated that the combination of $16 \mathrm{~S}$ rDNA and wsp PCR detection increased the detection efficiency and supergroup differentiation whereas detection with $f t s Z$ was less sensitive which can lead to falsenegative for Wolbachia infection mosquitoes.

Thus, this study aimed to determine natural Wolbachia infections in different field-collected mosquitoes from several states in Peninsular Malaysia and East Malaysia (Sabah) using Wolbachia $16 S$ rRNA and wsp gene amplifications, to determine the extent of natural infection of Wolbachia in some of these disease vectors and to understand its role in the dynamics of disease transmission.

\section{Methods \\ Mosquito collection}

Adult Anopheles spp. and Culicinae mosquitoes collected from 2013 to 2019 in Selangor, Kuala Lumpur, Putrajaya, Johor, Perak and Sabah (Fig. 1) using human landing catch, mosquito magnet trap and sticky trap, were randomly selected and screened for Wolbachia (Additional file 1: Table S1). All specimens were identified morphologically using the taxonomic keys of Reid [56], Sallum et al. [57] (Leucosphyrus group of Anopheles mosquitoes) and Jeffery et al. [58] prior to molecular detection of Wolbachia. Specimens which could not be reliably distinguished based on morphology, such as those of the Leucosphyrus group of Anopheles or those which were not well-preserved, were all molecularly identified by PCR and sequencing of the internal transcribed spacer 

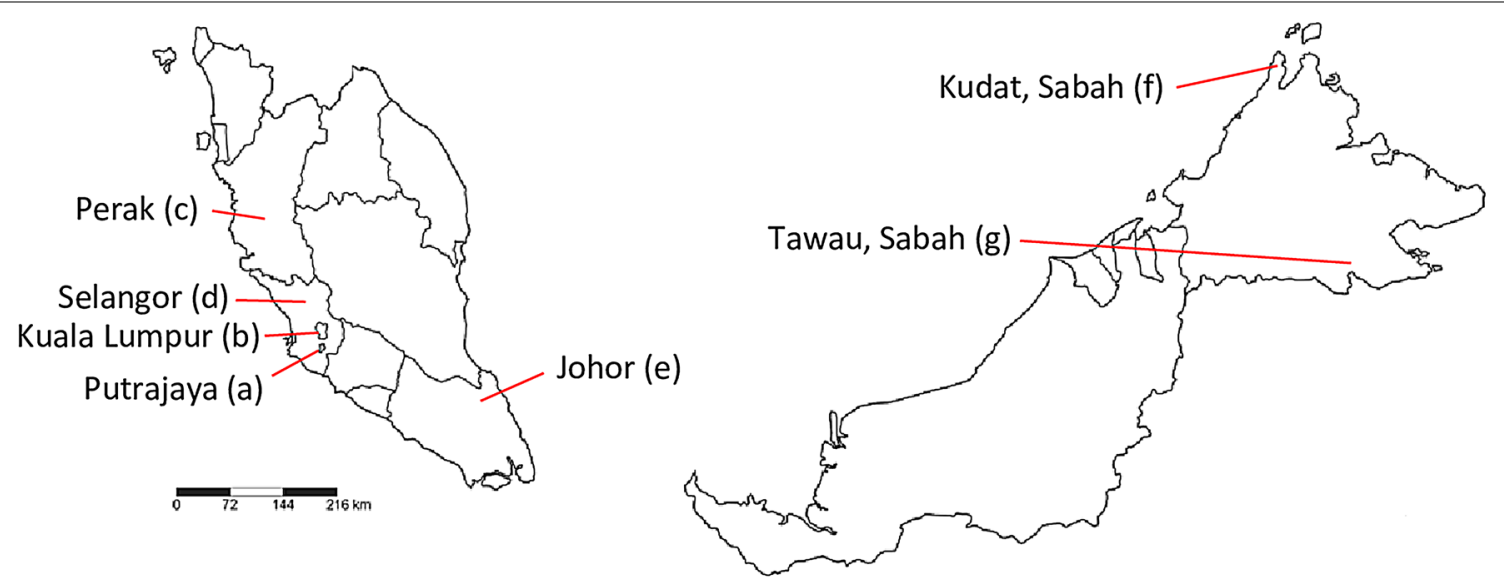

a

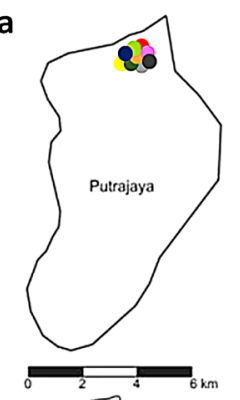

b
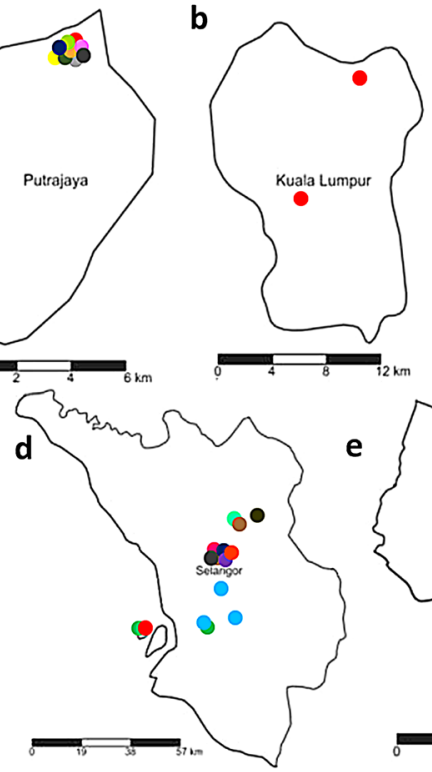
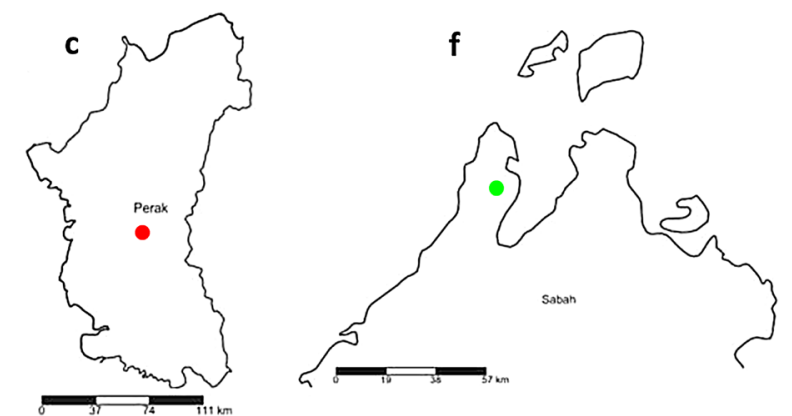

g
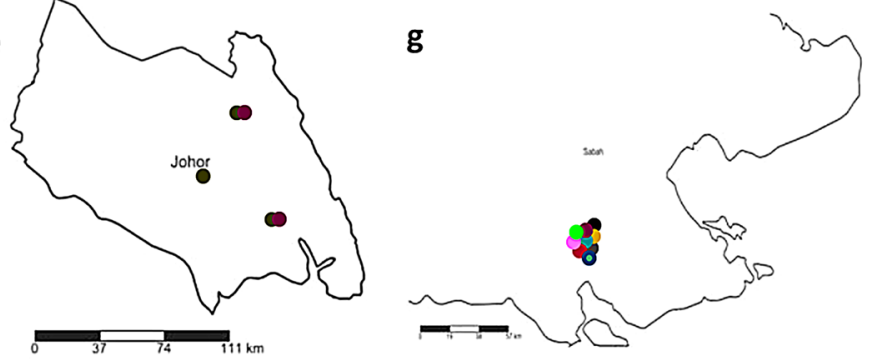

Anopheles (Cellia) group

- Anopheles balabacensis

- Anopheles introlatus

- Anopheles macarthuri

- Anopheles latens

- Anopheles karwari

- Anopheles kochi

- Anopheles maculatus

- Anopheles aconitus
Anopheles vagus

Anopheles watsonii

- Anopheles barbirostris

- Anopheles umbrosus

- Anopheles hyrcanus

- Anopheles sinensis

- Anopheles separatus

- Anopheles lindesayi group

Fig. 1 Map showing mosquitoes collected from five different states in Malaysia. a Putrajaya. b Kuala Lumpur. c Perak. d Selangor. e Johor. f Kudat, Sabah. g Tawau, Sabah 
2 (ITS2) gene [59]. If specimens could not be identified morphologically or molecularly, they were classified to the lowest level of taxonomic certainty, for example the genus level.

Additionally, Anopheles samples positive for Plasmodium infections were also included in the study (Additional file 1: Table S2). These 34 samples originating from other vector studies had already been screened for simian Plasmodium parasites (i.e. Plasmodium knowlesi, Plasmodium cynomolgi, Plasmodium inui, Plasmodium fieldi and Plasmodium coatneyi) infection by dissection and observation of the midgut for oocysts and salivary glands for sporozoites followed by PCR [60] in well-preserved samples; or by PCR of whole mosquitoes, head/thorax or abdomen in all other samples. The midguts were stained with $1 \%$ merchurochrome for visualization of oocysts, while the salivary glands were squashed in saline and viewed under a light compound microscope at $400 \times$ magnification for visualization of sporozoites.

\section{Genomic DNA extraction and molecular detection of Wolbachia}

Genomic DNA was extracted from the whole mosquito using InstaGene matrix (Bio-Rad, Hercules, California, USA) according to the manufacturer's protocol. The extracted DNA was run on a $1 \%$ agarose gel electrophoresis to confirm its presence. Detection of Wolbachia was undertaken targeting two conserved Wolbachia genes, wsp and $16 S$ rRNA. Wolbachia in Culicinae mosquitoes was screened using general $w s p$ primers (wsp81F: 5'-TGG TCC AAT AAG TGA TGA AGA AAC-3'; wsp691R: 5'-AAA AAT TAA ACG CTA CTC CA-3'), amplified in a $30 \mu \mathrm{l}$ reaction volume with $5 \mu \mathrm{l}$ DNA as template according to the standard PCR protocol [61]. These primers amplify a DNA fragment ranging from 590 to $632 \mathrm{bp}$. Nested PCR amplifying the $16 S$ rRNA gene was used to detect Wolbachia in Anopheles and Culicinae mosquitoes whose results were negative in the wsp PCR. The initial PCR employed $16 S$ Wolbachia-specific primers (W-Specf: 5'-CAT ACC TAT TCG AAG GGA TAG-3'; W-Specr: 5'-AGC TTC GAG TGA AAC CAA TTC- $3^{\prime}$ ) and was performed in $30 \mu$ reaction volume using $5 \mu \mathrm{l}$ DNA as template according to standard protocol in Werren \& Windsor [62]. Then, two microliters of the initial PCR products were amplified in a $30-\mu \mathrm{l}$ PCR reaction using specific internal primers (16SNF: 5'-GAA GGG ATA GGG TCG GTT CG-3'; 16SNR: 5'-CAA TTC CCA TGG CGT GAC G-3') as described in Shaw et al. [39]. The nested $16 S$ rRNA Wolbachia PCR generates a 412-bp DNA fragment. Aedes albopictus (Skuse) was used as positive control for all Wolbachia screening along with negative control (double-distilled water as template). All PCR products were run on $1.5 \%$ agarose gel electrophoresis and viewed under UV light prior to Sanger-sequencing by a commercial laboratory (MyTACG, Kuala Lumpur, Malaysia).

\section{Phylogenetic analysis}

The nucleotide sequences obtained were aligned using Geneious 9.1.6 software (http://www.geneious.com). All aligned Wolbachia sequences were compared with other sequences available in the GenBank database to determine the percentage identity using BLAST (http:// blast.ncbi.nlm.nih.gov/Blast.cgi) and the most similar sequences were downloaded for phylogenetic analysis. The alignment of all available sequences was examined visually and only those residues where variations were observed in sequences obtained from independent PCR amplifications from the same sample or from another independently amplified sample were considered reliable and therefore included in the phylogenetic analysis. All sequences ( $w s p=606$ nucleotides; $16 S$ rRNA $=374$ nucleotides) were exported to MEGA 7.0 software for further alignment and analysis using Clustal W algorithm [63]. Phylogenetic tree for the wsp gene was constructed using the Maximum Likelihood (best-fit substitution model) method. The phylogenetic tree model with lowest Bayesian information criterion (BIC) is considered to describe the substitution pattern the best. Hence, Tamura 3-parameter model was chosen for wsp gene analysis. Wsp gene sequence from Brugia pahangi was incorporated as the outgroup to confirm the outcome of the phylogenetic tree. For $16 S$ rRNA gene, the evolutionary history was inferred using the Maximum Likelihood method (best-fit substitution model). Based on the lowest BIC value, Jukes-Cantor model was chosen to infer the phylogenetic relationship of Wolbachia using the $16 \mathrm{~S}$ rRNA gene. The $16 S$ rRNA gene sequence of Rickettsia japonica was included as the outgroup. Both phylogeny tests were performed by bootstrap method with 1000 replications. All the evolutionary analyses were performed in MEGA 7.0 software. All sequences used in the phylogenetic analyses were submitted in the GenBank database under the accession numbers MN893348-MN893366 (wsp gene) and MN887537-MN887588 (16S rRNA).

\section{Results}

\section{Detection of Wolbachia in mosquitoes}

A total of 217 adult mosquitoes representing 26 mosquito species belonging to 5 genera (Anopheles, Culex, Aedes, Mansonia and Armigeres) (Additional file 1: Table S1) were screened for Wolbachia. These mosquitoes were collected from a wide range of habitats such as urban, island and forest (Additional file 1: Table S2). Polymerase chain reaction amplifying the Wolbachia wsp gene was successful in most of the Culicinae 
mosquito samples except for Culex vishnui, Culex pseudovishnui, Mansonia mosquitoes and some samples of Culex quinquefasciatus. Wolbachia infection was detected in these samples as well as in Anopheles spp. mosquitoes using the $16 S$ rRNA primers.

The overall Wolbachia infection rate for all mosquitoes tested in this study was $46.1 \%$. Wolbachia was detected in 4 species of mosquitoes (40/217) using wsp gene amplification and 15 species of mosquitoes $(60 / 217)$ using $16 S$ rRNA gene amplification (Table 1). The prevalence of Wolbachia infection within mosquito species ranged from $35.3 \%(6 / 17)$ in Cx. quinquefasciatus to $100 \%(19 / 19)$ in Ae. albopictus for wsp gene. Wolbachia infection rate by detection of Wolbachia $16 S$ rRNA gene ranged between $50-100 \%$ for $C x$. sinensis, Cx. vishnui, Cx. pseudovishnui and Mansonia mosquitoes. For Anopheles mosquitoes, the infection rate of Wolbachia among each species ranged between 21-57.1\%.

Presence of Wolbachia in different mosquito species from each state is given in Fig. 2 ( $w s p$ gene) and Fig. 3 (16S rRNA). While the prevalence appeared to be higher in certain states such as Selangor, this bias could be caused by the prevalence of Wolbachia in Ae. albopictus or higher number of mosquito species collected from Selangor (Fig. 3). Thus, it would be more valid if comparisons were made among mosquito species found in more than one site and are available in similar numbers, without taking Ae. albopictus into account. For example, the prevalence of Wolbachia was higher in An. barbirostris from Sabah than from Putrajaya, and the prevalence of Wolbachia was also higher in An. hyrcanus from Selangor than from Putrajaya. Similarly, the same scenario applies to the prevalence of Wolbachia in the mosquitoes according to ecological types (Table 2),

Table 1 Wolbachia infection prevalence rates in adult female mosquitoes using two conserved Wolbachia genes (16S rRNA and wsp)

\begin{tabular}{|c|c|c|c|c|c|}
\hline \multirow[t]{2}{*}{ Mosquito species } & \multirow[t]{2}{*}{$n$} & \multicolumn{2}{|c|}{ PCR-positive } & \multicolumn{2}{|c|}{ Prevalence $(95 \% \mathrm{Cl})(\%)^{\mathrm{a}}$} \\
\hline & & wsp & 165 rRNA & wsp & 16S rRNA \\
\hline Anopheles (Cellia) group & 1 & 0 & 0 & 0 & 0 \\
\hline An. balabacensis & 19 & 0 & 4 & 0 & $21.1(7.0-46.1)$ \\
\hline An. introlatus & 54 & 0 & 17 & 0 & $31.5(20.0-45.7)$ \\
\hline An. macarthuri & 4 & 0 & 1 & 0 & $25.0(1.3-78.1)$ \\
\hline An. latens & 8 & 0 & 4 & 0 & $50.0(17.5-82.6)$ \\
\hline An. karwari & 2 & 0 & 0 & 0 & 0 \\
\hline An. kochi & 1 & 0 & 0 & 0 & 0 \\
\hline An. maculatus & 9 & 0 & 2 & 0 & $22.2(3.9-59.8)$ \\
\hline An. vagus & 3 & 0 & 0 & 0 & 0 \\
\hline An. watsonii & 5 & 0 & 0 & 0 & 0 \\
\hline An. barbirostris & 10 & 0 & 5 & 0 & $50.0(20.1-79.9)$ \\
\hline An. umbrosus & 6 & 0 & 0 & 0 & 0 \\
\hline An. hyrcanus & 18 & 0 & 9 & 0 & $50.0(26.8-73.2)$ \\
\hline An. sinensis & 7 & 0 & 4 & 0 & $57.1(20.2-88.2)$ \\
\hline An. lindesayi species group & 1 & 0 & 0 & 0 & 0 \\
\hline An. separatus & 1 & 0 & 0 & 0 & 0 \\
\hline An. aconitus & 1 & 0 & 0 & 0 & 0 \\
\hline Cx.vishnui & 1 & 0 & 1 & 0 & $100(5.5-100)$ \\
\hline Cx.pseudovishnui & 2 & 0 & 2 & 0 & $100(19.8-100)$ \\
\hline Cx. quinquefasciatus & 17 & 6 & 5 & $35.3(15.3-61.4)$ & $29.4(11.4-56.0)$ \\
\hline Cx. sinensis & 2 & 0 & 1 & 0 & $50.0(2.7-97.3)$ \\
\hline Ma. annulifera & 1 & 0 & 1 & 0 & $100(5.5-100)$ \\
\hline Ma.bonneae & 1 & 0 & 1 & 0 & $100(5.5-100)$ \\
\hline Ar. subalbatus & 22 & 13 & 0 & $59.1(36.7-78.5)$ & 0 \\
\hline Ae. aegypti & 2 & 2 & 0 & $100(19.8-100)$ & 0 \\
\hline Ae. albopictus & 19 & 19 & 3 & $100(79.1-100)$ & $15.8(4.2-40.5)$ \\
\hline
\end{tabular}

Note: An individual was considered Wolbachia-infected when any one of the two Wolbachia gene fragments were amplified

Abbreviation: $\mathrm{Cl}$, confidence interval

${ }^{\text {a }}$ Wolbachia prevalence rate $(\%)=[$ No. of Wolbachia positive mosquitoes/Total no. of mosquitoes $] \times 100$ 


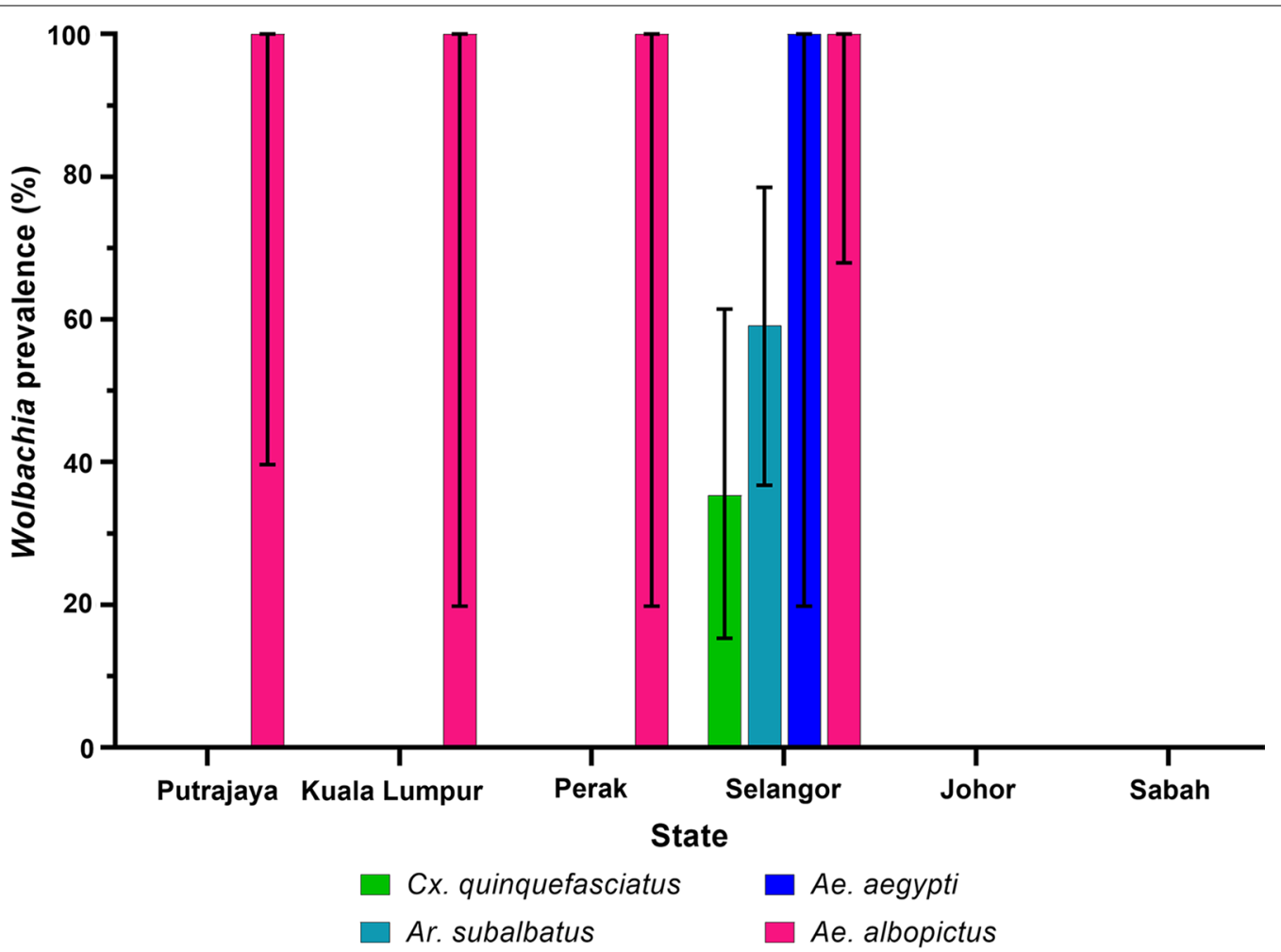

Fig. 2 Prevalence of Wolbachia-infected mosquitoes collected from different states in Malaysia using wsp primers. Error bar denotes 95\% confidence interval (Cl)

as most mosquitoes were collected from forested areas. Nonetheless, none of the An. balabacensis from the Banggi Island was found to be infected with Wolbachia, compared to infections found in the same species from mainland forested area. As for An. hyrcanus, $40 \%$ of the mosquitoes from the wetland were infected compared to $50-75 \%$ from the forested area. Thus, mosquitoes from forested areas, may have higher prevalence of Wolbachia compared to those from the wetland (Putrajaya) or island (Banggi Island) areas.

\section{Plasmodium-positive Anopheles with Wolbachia infection} Out of the 34 Plasmodium-positive Anopheles samples, 14 (38.24\%) were also found to have Wolbachia infection (Table 3). Interestingly, only 1 out of 9 (11.11\%) samples with oocysts/Plasmodium DNA-positive abdomens were positive for Wolbachia, as compared to 4 out of 8 (50\%) samples with sporozoites/Plasmodium DNA-positive head/thorax which were also Wolbachia-infected. However, this difference was not significant (Fisher's exact test, two-tailed, $P=0.131$ ). There was also no particular difference in Wolbachia prevalence between the small number of Plasmodium-positive Anopheles species investigated in this study.

\section{Phylogenetic analysis of Wolbachia in mosquitoes}

Eight wsp Wolbachia sequences from this study were deemed suitable for phylogenetic analysis after analysis of the sequencing results. We retrieved an additional $14 \mathrm{wsp}$ sequences and 1 outgroup sequence (B. pahangi) from GenBank for phylogenetic analysis. In total, 23 sequences were used for construction of the phylogenetic tree. The alignments for different species of mosquitoes are shown in Additional file 2: Figure S1 (Supergroup A) and Additional file 3: Figure S2 (Supergroup B). Phylogenetic tree of wsp gene inferred using ML method based on Tamura 3-parameter model (lowest $\mathrm{BIC}$ value $=2502.136$ ) resulted in two major clades belonging to Supergroups A and B with bootstrap values of $99 \%$ and $100 \%$, respectively (Fig. 4). Within the Wolbachia Supergroup B, two sub-clades branched out, one comprised of $w$ AlbB sequences from Ae. albopictus and Ae. aegypti (bootstrap value $=95 \%$ ) whereas Wolbachia from Cx. quinquefasciatus were grouped with $w$ Pip from $C x$. pipiens (bootstrap value $=100 \%$ ).

A total of $5216 \mathrm{~S}$ rRNA Wolbachia sequences were deemed suitable for use in phylogenetic analysis after analysing the sequencing results. Confirmation of $\mathrm{Wol}$ bachia supergroup for each sequence was executed using BLAST. All the sequences showed 99-100\% 


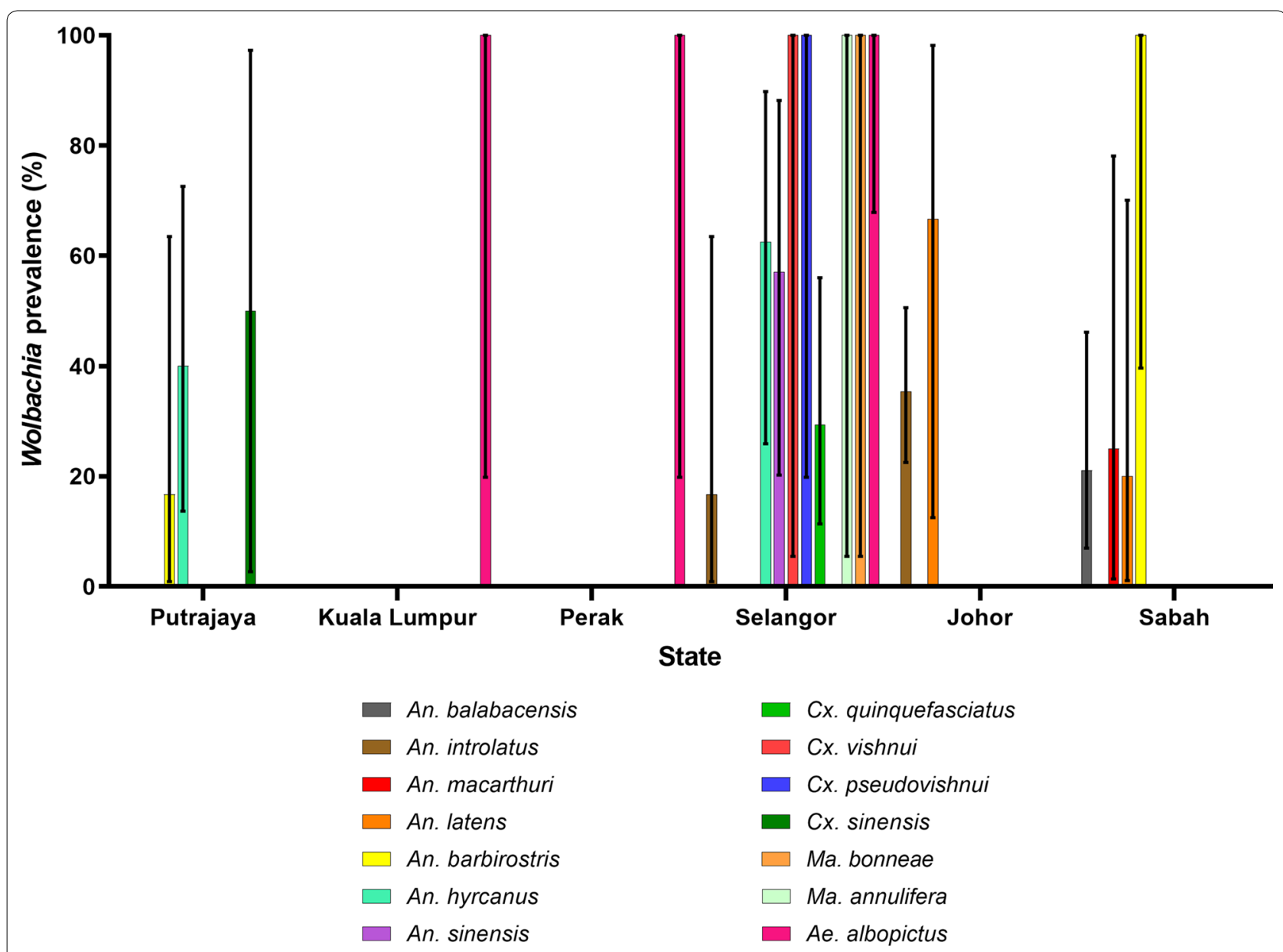

Fig. 3 Prevalence of Wolbachia-infected mosquitoes collected from different states in Malaysia using 16S rRNA primers. Error bar denotes $95 \%$ confidence interval (Cl)

nucleotide identities. Additionally, 26 sequences from GenBank were retrieved for phylogenetic analysis including 1 outgroup sequence ( $R$. japonica). Overall, 84 sequences were used for construction of Wolbachia $16 S$ rRNA phylogenetic tree. The alignments of $16 \mathrm{~S}$ rRNA sequences are shown in Additional file 4: Figure S3 (Supergroup A) and Additional file 5: Figure S4 (Supergroup B). The phylogenetic tree inferred using Maximum Likelihood method based on Jukes-Cantor model (lowest BIC value $=1897.45$ ) resulted in two major clades with high bootstrap value: Supergroup A (bootstrap value $=91 \%$ ) and Supergroup B (bootstrap value $=85 \%$ ) (Fig. 5). Within the Supergroup B clade, three sub-clades branched out. The first sub-clade consists of the majority of the Wolbachia sequences obtained from Anopheles mosquitoes (82.9\%) from this study and Wolbachia sequences from An. gambiae, Ae. albopictus and Ae. aegypti retrieved from GenBank (Fig. 5). Wolbachia 165 rRNA gene amplified from adults of $C x$. quinquefasciatus were clustered within the second sub-clade of Wolbachia Supergroup B, confirming the phylogenetic relationship inferred using wsp gene. A single sample of each Ma. annulifera and Ma. bonneae was found infected with Wolbachia using $16 S$ rRNA gene. Only Wolbachia-infected Ma. annulifera sequence was obtained in this study and it was grouped together in the Supergroup B with $w$ Unif-Mad isolated from Ma. uniformis, as well as Wolbachia from Cx. quinquefasciatus and Anopheles mosquitoes from Kayin state, Myanmar. Repeated attempts to sequence Wolbachia 16s rRNA gene from Wolbachia-infected $M a$. bonneae failed to provide reliable sequences and thus were not available. The third sub-clade comprised Wolbachia sequences from Anopheles mosquitoes found in Myanmar. On the other hand, Wolbachia from two Culicinae and seven Anopheles spp. mosquitoes were grouped with Wolbachia Supergroup A.

While investigating if any strains of Wolbachia was associated with a particular species of mosquito, Wolbachia from Armigeres subalbatus and the only one 
Table 2 Wolbachia infection prevalence rates in adult female mosquitoes in different ecological types across the states in Malaysia

\begin{tabular}{|c|c|c|c|}
\hline \multirow[t]{2}{*}{ Ecological type/study site } & \multirow[t]{2}{*}{ Mosquito species } & \multicolumn{2}{|c|}{ Prevalence (95\% Cl) (\%) } \\
\hline & & wsp & 165 rRNA \\
\hline \multicolumn{4}{|l|}{ Urban } \\
\hline Bangsar, Kuala Lumpur & Ae. albopictus & $100(5.5-100)$ & - \\
\hline Jalan Genting, Kuala Lumpur & & $100(5.5-100)$ & $100(5.5-100)$ \\
\hline Pasir Puteh, Perak & & $100(19.8-100$ & $100(19.8-100)$ \\
\hline Damansara Damai, Selangor & Ae. aegypti & $100(19.8-100)$ & - \\
\hline Persanda, Selangor & Cx.quinquefasciatus & $42.9(11.8-79.8)$ & $28.6(5.1-69.8)$ \\
\hline Klang, Selangor & & $30.0(8.1-64.6)$ & $30.0(8.1-64.6)$ \\
\hline \multicolumn{4}{|l|}{ Island } \\
\hline Pulau Ketam, Selangor & Ae. albopictus & $100(39.6-100)$ & - \\
\hline Pulau Banggi, Sabah & An. balabacensis & 0 & 0 \\
\hline \multicolumn{4}{|l|}{ Wetland } \\
\hline \multirow[t]{10}{*}{ Putrajaya } & An. hyrcanus & 0 & $40.0(13.7-72.6)$ \\
\hline & An. (Cellia) group & 0 & 0 \\
\hline & An. barbirostris & 0 & $20.0(1.1-70.1)$ \\
\hline & An. umbrosus & 0 & 0 \\
\hline & An. maculatus & 0 & 0 \\
\hline & An. karwari & 0 & 0 \\
\hline & An. separatus & 0 & 0 \\
\hline & An. lindesayi group & 0 & 0 \\
\hline & Cx. sinensis & 0 & $50.0(2.7-97.3)$ \\
\hline & Ae. albopictus & 100 & - \\
\hline \multicolumn{4}{|l|}{ Forest } \\
\hline \multirow[t]{3}{*}{ Ulu Kalong, Selangor } & An. introlatus & 0 & $16.7(0.9-63.5)$ \\
\hline & An. maculatus & 0 & $100(19.8-100)$ \\
\hline & Ar. subalbatus & $59.1(36.7-78.5)$ & - \\
\hline \multirow[t]{3}{*}{ Ulu Kalong, Selangor } & Ma.bonneae & 0 & $100(5.5-100)$ \\
\hline & Ae. albopictus & $100(56.1-100)$ & - \\
\hline & Culex. sp. & $100(5.5-100)$ & - \\
\hline \multirow[t]{5}{*}{ Bukit Lagong, Selangor } & An. maculatus & 0 & 0 \\
\hline & An. hyrcanus & 0 & $75.0(21.9-98.7)$ \\
\hline & Ma. annulifera & 0 & $100(5.5-100)$ \\
\hline & Cx.vishnui & 0 & $100(5.5-100)$ \\
\hline & Cx.pseudovishnui & 0 & $100(5.5-100)$ \\
\hline \multirow[t]{2}{*}{ Sg. Sendat, Selangor } & An. sinensis & 0 & $57.1(20.2-88.2)$ \\
\hline & An. hyrcanus & 0 & $50.0(9.2-90.8)$ \\
\hline \multirow[t]{9}{*}{ Tawau, Sabah } & An. vagus & 0 & 0 \\
\hline & An. maculatus & 0 & 0 \\
\hline & An. watsonii & 0 & 0 \\
\hline & An. funestus & 0 & 0 \\
\hline & An. kochi & 0 & 0 \\
\hline & An. macarthuri & 0 & $25.0(1.3-78.1)$ \\
\hline & An. latens & 0 & $20.0(1.1-70.1)$ \\
\hline & An. balabacensis & 0 & $23.5(7.8-50.2)$ \\
\hline & An. barbirostris & 0 & $100(39.6-100)$ \\
\hline Kluang, Johor & An. introlatus & 0 & $55.6(22.7-84.7)$ \\
\hline \multirow[t]{2}{*}{ Mersing, Johor } & An. introlatus & 0 & $24.2(11.7-42.6)$ \\
\hline & An. latens & 0 & $100(5.5-100)$ \\
\hline \multirow[t]{3}{*}{ Kota Tinggi, Johor } & An. introlatus & 0 & $50.0(14.0-86.1)$ \\
\hline & An. latens & 0 & $50.0(2.7-97.3)$ \\
\hline & An. umbrosus & 0 & 0 \\
\hline
\end{tabular}


Table 2 (continued)

Abbreviation: $\mathrm{Cl}$, confidence interval

Table 3 Plasmodium-positive Anopheles with Wolbachia infection

\begin{tabular}{|c|c|c|c|c|}
\hline Species & No. of samples & No. of samples: stage/type of infection & $\begin{array}{l}\text { No. of Wolbachia-positive samples/No. of } \\
\text { samples }\end{array}$ & $\begin{array}{l}\text { No. of samples positive } \\
\text { for Wolbachia/Total no. of } \\
\text { samples }\end{array}$ \\
\hline An. introlatus & 28 & $\begin{array}{l}\text { 17: Plasmodium DNA-positive whole } \\
\text { mosquito; } \\
\text { 4: oocysts/Plasmodium DNA-positive } \\
\text { abdomen; } \\
\text { 7: sporozoites/Plasmodium DNA-positive } \\
\text { head/thorax }\end{array}$ & $\begin{array}{l}\text { 8/17 whole mosquito samples; } \\
\text { 0/4 samples with oocysts/Plasmodium } \\
\text { DNA-positive abdomen; } \\
\text { 4/7 samples with sporozoites/Plasmodium } \\
\text { DNA-positive head/thorax }\end{array}$ & $12 / 28$ \\
\hline An. latens & 2 & $\begin{array}{l}\text { 1: oocysts; } \\
\text { 1: Plasmodium DNA-positive abdomen }\end{array}$ & $\begin{array}{l}\text { 1/1 sample with oocysts; } \\
\text { 0/1 sample with Plasmodium DNA-posi- } \\
\text { tive abdomen }\end{array}$ & $1 / 2$ \\
\hline An. balabacensis & 2 & $\begin{array}{l}\text { 1: sporozoites; } \\
\text { 1: oocysts }\end{array}$ & 0 & $0 / 2$ \\
\hline An. umbrosus & 2 & 2: oocysts & 0 & $0 / 2$ \\
\hline Total & 34 & $\begin{array}{l}\text { 17: whole mosquitoes; } \\
\text { 9: oocysts/Plasmodium DNA-positive } \\
\text { abdomen } \\
\text { 8: sporozoites/Plasmodium DNA-positive } \\
\text { head/thorax }\end{array}$ & $\begin{array}{l}\text { 8/17 whole mosquito samples; } \\
\text { 1/9 samples with oocysts/Plasmodium } \\
\text { DNA-positive abdomen; } \\
\text { 4/8 samples with sporozoites/Plasmodium } \\
\text { DNA-positive head/thorax }\end{array}$ & $13 / 34$ \\
\hline
\end{tabular}

sample from Cx. vishnui were all found to be grouped in Supergroup A, whereas Wolbachia from $C x$. quinquefasciatus mosquitoes were of Supergroup B. Other than these, Wolbachia from other mosquito species belonged to either supergroups, although most of them were grouped in Wolbachia Supergroup B.

\section{Discussion}

In our study, we assessed the prevalence of Wolbachia in mosquito species collected from Peninsular Malaysia and Sabah. To our knowledge, this study is the first report of natural Wolbachia infections in field adult mosquito populations in Malaysia detected using Wolbachia wsp and $16 S$ rRNA PCR amplifications. Our study further supports the higher sensitivity of the $16 S$ rRNA nested PCR described by Shaw et al. [39] over the standard wsp PCR from Zhou et al. [61] for the detection of Wolbachia in mosquitoes due to genetic divergence or weak infection density [44]. The overall infection rate of all tested mosquitoes in this study was $46.1 \%$. Wolbachia infection rates of $28.1 \%$ and $37.8 \%$ have been previously reported in the natural mosquito populations from neighboring countries Singapore [3] and Thailand [8], respectively.

The nested PCR utilizing $16 S$ rRNA primers detected Wolbachia in seven species of Anopheles (41/144; 28.5\%), i.e. An. balabacensis, An. latens, An. introlatus, An. macarthuri (Leucosphyrus Group), An. barbirostris (Barbirostris Group), An. hyrcanus and An. sinensis (Hyrcanus
Group). Of these, An. latens [64], An. introlatus [59] and $A n$. balabacensis [65] of the Leucosphyrus Group were incriminated as vectors of Plasmodium knowlesi in Malaysia. Additionally, An. balabacensis is also a vector of human malaria and Bancroftian filariasis in Sabah, Malaysia [66]. Other Anopheles species such as An. barbirostris [67] and An. sinensis [68] are vectors of human malaria, whereas An. hyrcanus has been postulated to be a vector for malaria due to its simultaneous occurrence with malaria cases [69]. These data along with the report of Wolbachia in natural mosquito populations in Kayin state, Myanmar [46] and Africa [38-40, 42-44], reinforce the occurrence of natural Wolbachia infections in malaria vectors.

Conclusive prevalence of Wolbachia based on different habitats cannot be drawn. This is due to the heterologous distribution of the mosquito species themselves and differences in number of mosquito species available between the sites. The prevalence appeared to be biased in certain states or environments mostly due to more mosquito species collected from the particular site than the others. However, there was a slight indication that Wolbachia infections can be more prevalent in forested areas than wetlands or island. This can be attributed to the richer flora and fauna in the forested areas which may have many more hosts with stable Wolbachia, which may facilitate horizontal transfers to other species. 


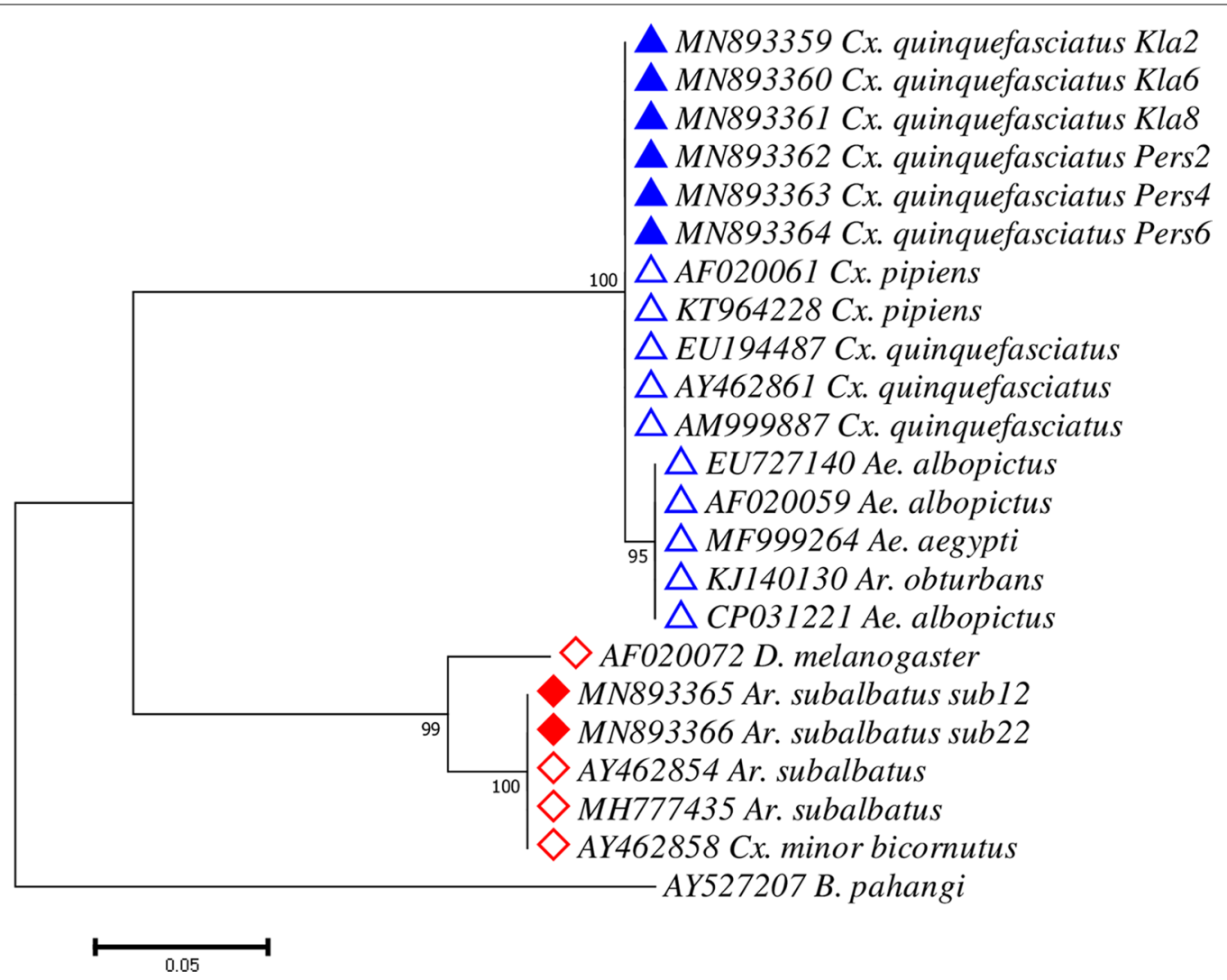

Fig. 4 Maximum Likelihood phylogenetic analysis for Wolbachia using the wsp gene. The tree with the lowest Bayesian Information Criterion (BIC) (2725.733879) is shown, incorporating 33 nucleotide sequences (8 sequences from this study; 14 sequences from GenBank) and one outgroup (B. pahangi). Wolbachia sequences of Supergroup A are denoted with red diamonds while those of Supergroup B are denoted with blue triangles. Sequence retrieved from GenBank is denoted with hollow diamond or triangle along with its respective accession number while sequence obtained from this study are denoted with a filled diamond or triangle

Molecular phylogeny based on $16 \mathrm{~S}$ rRNA sequences revealed that most Wolbachia infecting Anopheles (34/41) clustered with $w$ AlbB (Supergroup B) isolated from Ae. albopictus [70], whereas the remaining Wolbachia strains from other Anopheles (7/41) were more closely related to $w \mathrm{Mel}$ (D. melanogaster) of Supergroup A. Interestingly, all the Wolbachia infecting Anopheles detected in this study were shown to be more closely related with those recently reported in African malaria vectors (wAnga) [38-40, 42-44] than with the ones from Myanmar [46] (Fig. 5). Although recent publications suggest a lack of concrete evidence regarding the presence of Wolbachia in Anopheles and Ae. aegypti [71, 72], our results show that Wolbachia is present in Anopheles. Our sequences were clean and matched those of Wolbachia Supergroup A and B. However, we agree that our limited study shows that the prevalence of Wolbachia in Anopheles is much lower than the natural presence of Wolbachia in Ae. albopictus.

Plasmodium-infected Anopheles mosquitoes were also found to be Wolbachia-infected. These mosquitoes harbored oocysts or sporozoites. Results from this study found that Wolbachia prevalence is higher (though not significant) in Anopheles mosquitoes with sporozoites (50\%) than in mosquitoes with oocysts (11.11\%). This is in agreement with a study finding significant reductions in $P$. berghei or $P$. falciparum oocyst infections in An. gambiae transiently and somatically-infected with $w$ MelPop or $w$ AlbB Wolbachia strains $[36,37]$. It was also found that natural populations of Wolbachiainfected An. coluzzii females have lower frequency of Plasmodium infections than Wolbachia-negative individuals. Although the study did not explicitly mention the infection stages of the parasite in these mosquitoes, it is assumed that most of these Plasmodium infections could be at the oocyst stage since Plasmodium DNA was detected from dissected abdomens and thoraces of the mosquitoes 5 days after indoor collection [39]. Another interesting study also showed that Culex pipiens (the 


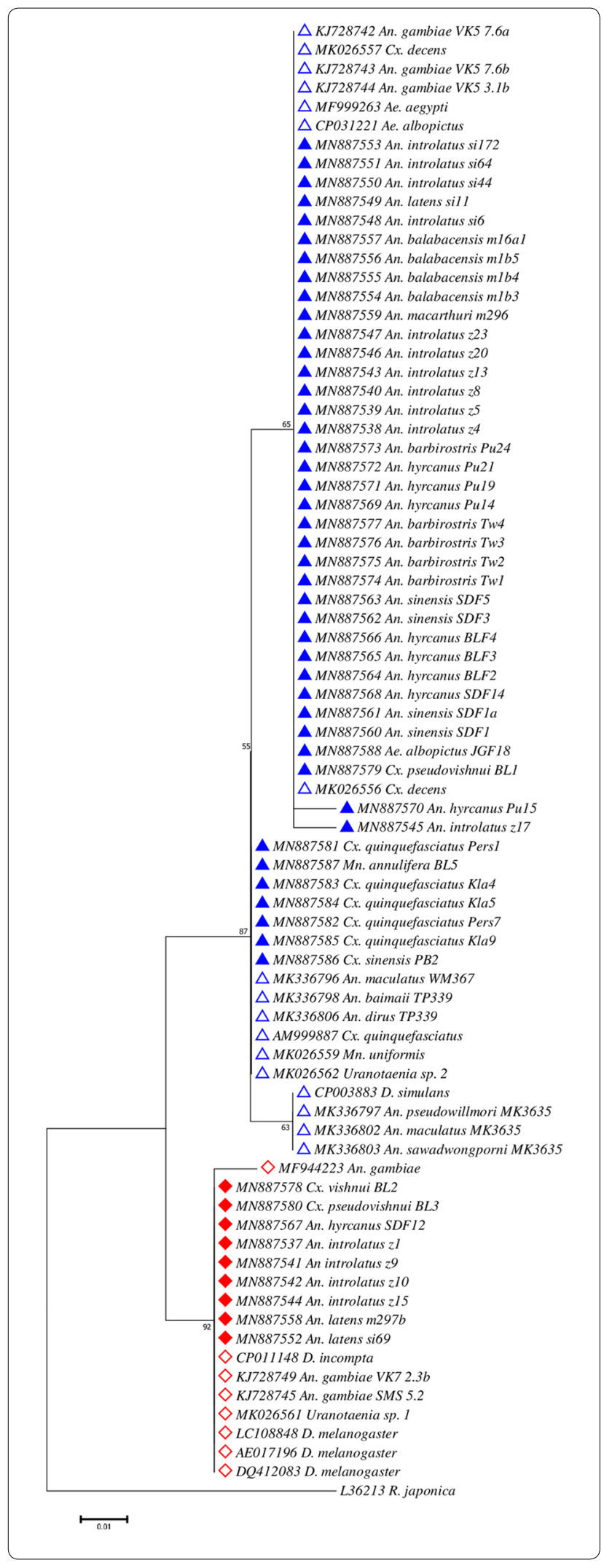

Fig. 5 Maximum Likelihood phylogenetic analysis for Wolbachia using the 165 rRNA gene. The tree with the lowest Bayesian Information Criterion (BIC) (2020.770483) is shown, incorporating 83 nucleotide sequences (52 sequences from this study; 31 sequences from GenBank) and one outgroup ( $R$. japonica). Wolbachia sequences of Supergroup $A$ are denoted with red diamonds while those of Supergroup B are denoted with blue triangles. Sequences retrieved from GenBank are denoted with an open diamond or triangle along with its respective accession number while sequences obtained from this study are denoted with a filled diamond or triangle

natural vector of $P$. relictum), naturally infected with Wolbachia, had increased sporozoites than those without Wolbachia [73]. Thus, it seems to demonstrate that when Wolbachia is naturally present in the mosquitoes, it may perturb oocysts development but promote sporozoites production. This however, is the opposite to the findings of a study on An. gambiae from Mali, Africa showing that Wolbachia infection was significantly lower in sporozoite-positive mosquitoes compared to the sporozoite-negative mosquitoes. However, Wolbachia-positive mosquitoes had higher oocyst infection compared to Wolbachia negative-mosquitoes [40]. It seems that Wolbachia can suppress the prevalence and intensity of P. falciparum sporozoite infections in wild An. gambiae [40]. On the other hand, An. stephensi experimentally infected with $w$ AlbB strain of Wolbachia was able to suppress both $P$. falciparum oocysts and sporozoite infection rates [34]. Due to these contrasting results, it is pertinent for us to study the life-cycle and transmission dynamics of Plasmodium in Anopheles vectors infected and uninfected with Wolbachia. It is possible that Wolbachia may affect only certain Plasmodium parasites present in the vector such as $P$. falciparum as reported by Bian et al. [34] or simian malaria parasites in this study, or it might influence vector competence of certain vector species [73]. As such, effect of Wolbachia on An. balabacensis, An. latens and An. introlatus in the present study remains obscure and hence, there is a need for extensive mosquito collections and thorough investigations.

Naturally, Ae. aegypti does not harbor Wolbachia. Interestingly, in this study the two Ae. aegypti which emerged from larvae collected from sticky traps were positive for Wolbachia. However, Wolbachia has been found in large percentage of Ae. aegypti in Manila [47], and in Kuala Lumpur, Malaysia, Wolbachia of Supergroup B has been reported from Ae. aegypti larvae which were also positive for DENV 2 virus [49]. Similarly, in India [50], Wolbachia has been detected in natural Ae. aegypti populations and was designated as $w$ AegB. Additionally, Wolbachia-infected Ae. aegypti has been 
reported from Mexico (>50\%) [52], Florida [51], Panama [54] and Texas [53]. It is noteworthy that during this study, no Wolbachia-infected mosquito colony is being reared in our insectarium, neither is there any routine screening nor handling of Wolbachia material.

The Wolbachia infecting Cx. quinquefasciatus was shown to be more closely related to $w$ Pip, isolated from Cx. pipiens within the Supergroup B than with $w$ AlbB from Ae. albopictus, similar to previous reports [4, 74]. Our results also revealed the presence of Wolbachia in Ar. subalbatus which was grouped within the Supergroup A. Interestingly, all $C x$. vishnui, $C x$. pseudovishnui, $C x$. sinensis (vector of Japanese encephalitis), Ma. annulifera, Ma. bonneae and some $C x$. quinquefasciatus mosquitoes failed to show a positive wsp PCR amplification. Through nested PCR of the $16 S$ rRNA gene, this is the first report of Wolbachia infection detected in Cx. sinensis, Cx. vishnui, Cx. pseudovishnui, Ma. bonneae and Ma. annulifera. However, due to the low number of samples collected, further studies are needed to determine if this is a result of strain variability or low-density infections which resulted in failure of amplification using $w s p$ primers.

While the PCR and sequencing results of the present study are convincing, especially by showing that the Wolbachia strains found infecting the mosquitoes belong to the two supergroups ( $\mathrm{A}$ and $\mathrm{B}$, which are associated with arthropods), there is a growing call for careful investigations into natural Wolbachia infections in previously unreported mosquitoes. This is because, it has been estimated that over half of terrestrial arthropod species are infected with Wolbachia [75]. Therefore, the environment is a factor that possibly affects the presence or absence of Wolbachia in some mosquitoes [43]. Thus, environmental contamination of the samples from plants, endo- and ectoparasites, Wolbachia-contaminated/infected food in the wild, eggs and larval habitats and other cohabitating insects cannot be ruled out [46, 71]. Furthermore, reports of Wolbachia found with low prevalence in some species and difficulty in detecting them, especially in Anopheles spp. [42-44, 46], indicate that there could be a lack of stable symbiotic relationship between Wolbachia and the hosts [71]. Therefore, to demonstrate evidence of a stable, intraovarially-transmitted Wolbachia symbiont in a host [71], steps that should be taken include: (i) visualizing Wolbachia in different host tissues using fluorescent in situ hybridization (FISH) or electron microscopy; (ii) demonstrating that the infection is and can be maternally transmitted by performing reciprocal crosses; and (iii) showing that Wolbachia can be removed from the mosquitoes by antibiotic or heat treatment [72].

Nonetheless, the growing reports of Wolbachia infections in previously unreported mosquito vectors, open avenues for further investigations into its prevalence and should prompt careful evaluations on the release of Wolbachia-infected mosquitoes for disease/vector control programmes. Additionally, the presence of Asaia, an acetic acid bacterium found in some Anopheles species is postulated for the absence of Wolbachia in Anopheles mosquitoes [76]. Asaia has been shown to impede vertical transmission of Wolbachia in Anopheles mosquitoes experimentally and found negatively related with Wolbachia in mosquito reproductive organs [77]. Therefore, the dynamics of Wolbachia infections with vector capacity, microbial interactions within the host and disease transmission require further in-depth studies.

\section{Conclusions}

The present study reports the presence of Wolbachia in Anopheles and Ae. aegypti which were not previously reported, as well as in other important mosquito vector genera such as Culex and Mansonia using $16 S$ rRNA and $w s p$ genes. Given the fact that Wolbachia can reduce the life span of its host, prevent pathogen from completing its life-cycle, as well as reduce susceptibility of host to pathogen infection, Wolbachia is being considered for vector control programmes and is now released on a large scale in many countries. Thus, it is pertinent to carry out large-scale studies on the natural infection of Wolbachia in mosquito vectors using more sensitive and accurate methods of detection as described above. The long-term effect of introduced Wolbachia into new hosts and its effect on pathogen suppression should also be studied.

\section{Supplementary information}

Supplementary information accompanies this paper at https://doi. org/10.1186/s13071-020-04277-x.

Additional file 1: Table S1. Summary of mosquito species used in the study from six states in Malaysia. Collection was carried out using human landing catch (HLC), mosquito magnetic trap and ovitrap. Table S2. Mosquito collection sites. Mosquitoes were collected from different settings: urban, village, island, forest and wetland across several states in Malaysia. Table S3. Anopheles samples with Plasmodium infections.

Additional file 2: Figure S1. Alignment and variable sites of Wolbachia wsp sequences (Supergroup A) with nucleotide positions.

Additional file 3: Figure S2. Alignment and variable sites of Wolbachia wsp sequences (Supergroup B) with nucleotide positions.

Additional file 4: Figure S3. Alignment and variable sites of Wolbachia 165 rRNA (Supergroup A) sequences with nucleotide positions.

Additional file 5: Figure S4. Alignment and variable sites of Wolbachia 165 rRNA (Supergroup B) sequences with nucleotide positions.

Abbreviations

wsp: Wolbachia surface protein; 165 rRNA: 165 ribosomal RNA. 


\section{Acknowledgements}

The authors thank the staff of vector control program for the help they provided during the collection of mosquitoes. The authors would also like to thank other colleagues who have volunteered to collect some of the mosquitoes and Dr. Francesco Baldini for Wolbachia DNA from An. arabiensis.

\section{Authors' contributions}

IV, WML and JWKL conceived and designed the study. WML, WKW, SP, WYWS, NKJ, CSL, VLL and NMH were responsible for the field work. WML, WKW, SP, NKY and NMH carried out laboratory work. WML, WKW, SP and JWKL carried out the molecular work. ML, VLL, JWKL and IV analysed the data and prepared the manuscript. All other authors were responsible for editorial processing of the manuscript. All authors read and approved the final manuscript.

\section{Funding}

This study was funded by Ministry of Education, Malaysia Long-term Research Grant Scheme LRGS 2018-1(LR002C-2018). The funding body has no role in the design of the study and collection, analysis and interpretation of data and in writing the manuscript.

\section{Availability of data and materials}

All data generated or analysed during this study are included in this published article and its additional files. The newly generated sequences were deposited in the GenBank database under the accession numbers MN893348-MN893366 (wsp gene) and MN887537-MN887588 (16S rRNA).

\section{Ethics approval and consent to participate}

This study was approved by the Medical Research and Ethics Committee (NMRR, Reference No. NMRR-12-786-13048 and NMRR 19-962-47606 (IIR)), for the collection of mosquitoes and registered in the National Medical Research Register of the Malaysian Ministry of Health.

\section{Consent for publication}

Not applicable.

\section{Competing interests}

The authors declare they have no competing interests.

\section{Author details}

${ }^{1}$ Department of Parasitology, Faculty of Medicine, University of Malaya, 50603 Kuala Lumpur, Malaysia. ${ }^{2}$ Department of Biomedical Science, Faculty of Medicine, University of Malaya, 50603 Kuala Lumpur, Malaysia. ${ }^{3}$ Entomology \& Pest Unit, Kluang District Health Office, 86000 Kluang, Johor, Malaysia. ${ }^{4}$ Tropical Infectious Diseases Research and Education Centre (TIDREC), University of Malaya, 50603 Kuala Lumpur, Malaysia.

Received: 14 April 2020 Accepted: 3 August 2020

Published online: 12 August 2020

\section{References}

1. Werren JH, Baldo L, Clark ME. Wolbachia: master manipulators of invertebrate biology. Nat Rev Microbiol. 2008;6:741-51.

2. Zug R, Hammerstein P. Still a host for Wolbachia: analysis of recent data suggests that $40 \%$ of terrestrial arthropod species are infected. PloS ONE. 2012; $7:$ e38544.

3. Kittayapong P, Baisley KJ, Baimai V, O'Neill SL. Distribution and diversity of Wolbachia infections in Southeast Asian mosquitoes (Diptera: Culicidae). J Med Entomol. 2000;37:340-5.

4. Nugapola N, De Silva W, Karunaratne S. Distribution and phylogeny of Wolbachia strains in wild mosquito populations in Sri Lanka. Parasit Vectors. 2017;10:230.

5. Raharimalala FN, Boukraa S, Bawin T, Boyer S, Francis F. Molecular detection of six (endo-) symbiotic bacteria in Belgian mosquitoes: first step towards the selection of appropriate paratransgenesis candidates. Parasitol Res. 2016;115:1391-9.
6. Rasgon JL, Scott TW. An initial survey for Wolbachia (Rickettsiales: Rickettsiaceae) infections in selected California mosquitoes (Diptera: Culicidae). J Med Entomol. 2004:41:255-7.

7. Soni M, Bhattacharya C, Sharma J, Khan SA, Dutta P. Molecular typing and phylogeny of Wolbachia: a study from Assam, north-eastern part of India. Acta Trop. 2017;176:421-6.

8. Wiwatanaratanabutr I. Geographic distribution of wolbachial infections in mosquitoes from Thailand. J Invertebr Pathol. 2013;114:337-40.

9. Yamada R, Iturbe-Ormaetxe I, Brownlie JC, O'Neill SL. Functional test of the influence of Wolbachia genes on cytoplasmic incompatibility expression in Drosophila melanogaster. Insect Mol Biol. 2011;20:75-85.

10. O'Neill SL, Giordano R, Colbert AM, Karr TL, Robertson HM. 165 rRNA phylogenetic analysis of the bacterial endosymbionts associated with cytoplasmic incompatibility in insects. Proc Natl Acad Sci USA. 1992;89:2699-702.

11. O'Neill SL, Hoffman A, Werren JH. Influential passengers: inherited microorganisms and arthropod reproduction. Oxford: Oxford University Press; 1997.

12. Werren JH. Biology of Wolbachia. Annu Rev Entomol. 1997;42:587-609.

13. Brelsfoard CL, Dobson SL. Wolbachia-based strategies to control insect pests and disease vectors. Asia-Pac J Mol Biol Biotechnol. 2009;17:55-63.

14. Turelli M, Hoffman AA. Rapid spread of an inherited incompatibility factor in California Drosophila. Nature. 1991;353:440-2.

15. Fraser JE, De Bruyne JT, Iturbe-Ormaetxe I, Stepnell J, Burns RL, Flores HA, et al. Novel Wolbachia-transinfected Aedes aegypti mosquitoes possess diverse fitness and vector competence phenotypes. PLoS Pathog. 2017;13:e1006751.

16. Clancy DJ, Hoffmann AA. Environmental effects on cytoplasmic incompatibility and bacterial load in Wolbachia-infected Drosophila simulans. Entomol Exp Appl. 1998:86:13-24.

17. Panteleev D, Goriacheva II, Andrianov BV, Reznik NL, Lazebnyi OE, Kulikov AM. The endosymbiotic bacterium Wolbachia enhances the nonspecific resistance to insect pathogens and alters behavior of Drosophila melanogaster. Genetika. 2007;43:1277-80.

18. Wiwatanaratanabutr I, Kittayapong P. Effects of crowding and temperature on Wolbachia infection density among life cycle stages of Aedes albopictus. J Invertebr Pathol. 2009;102:220-4.

19. Wiwatanaratanabutr S, Kittayapong P. Effects of temephos and temperature on Wolbachia load and life history traits of Aedes albopictus. Med Vet Entomol. 2006;20:300-7.

20. Iturbe-Ormaetxe I, Walker T, O'Neill SL. Wolbachia and the biological control of mosquito-borne disease. EMBO Rep. 2011;12:508-18.

21. Joubert DA, Walker T, Carrington LB, De Bruyne JT, Kien DH, Hoang Nle T, et al. Establishment of a Wolbachia superinfection in Aedes aegypti mosquitoes as a potential approach for future resistance management. PloS Pathog. 2016;12:e1005434.

22. Bian G, Xu Y, Lu P, Xie Y, Xi Z. The endosymbiotic bacterium Wolbachia induces resistance to dengue virus in Aedes aegypti. PloS Pathog. 2010;6:e1000833.

23. Frentiu FD, Zakir T, Walker T, Popovici J, Pyke AT, Van den Hurk AF, et al. Limited dengue virus replication in field-collected Aedes aegypti mosquitoes infected with Wolbachia. PloS Negl Trop Dis. 2014;8:e2688.

24. Joanne S, Vythilingam I, Teoh B-T, Leong C-S, Tan K-K, Wong M-L, et al. Vector competence of Malaysian Aedes albopictus with and without Wolbachia to four dengue virus serotypes. Trop Med Int Health. 2017;22:1154-65.

25. Moreira LA, Iturbe-Ormaetxe I, Jeffery JA, Lu G, Pyke AT, Hedges LM, et al. A Wolbachia symbiont in Aedes aegypti limits infection with dengue, chikungunya and Plasmodium. Cell. 2009;139:1268-78.

26. Mousson L, Zouache K, Arias-Goeta C, Raquin V, Mavingui P, Failloux AB. The native Wolbachia symbionts limit transmission of dengue virus in Aedes albopictus. PloS Negl Trop Dis. 2012;6:e1989.

27. Walker T, Johnson PH, Moreira LA, Iturbe-Ormaetxe I, Frentiu FD, McMeniman CJ, et al. The wMel Wolbachia strain blocks dengue and invades caged Aedes aegypti populations. Nature. 2011;476:450-3.

28. Xi Z, Khoo CC, Dobson SL. Wolbachia establishment and invasion in an Aedes aegypti laboratory population. Science. 2005;310:326-8. 
29. Ahmad NA, Vythilingam I, Lim YAL, Zabari NZAM, Lee HL. Detection of Wolbachia in Aedes albopictus and their effects on chikungunya virus. Am J Trop Med Hyg. 2017;96:148-56.

30. Van den Hurk AF, Hall-Mendelin S, Pyke AT, Frentiu FD, McElroy K, Day A, et al. Impact of Wolbachia on infection with chikungunya and yellow fever viruses in the mosquito vector Aedes aegypti. PloS Negl Trop Dis. 2012;6:e1892.

31. Aliota MT, Peinad SA, Velez ID, Osorio JE. The wMel strain of Wolbachia reduces transmission of Zika virus by Aedes aegypti. Sci Rep. 2016;6:28792.

32. Chouin-Carneiro T, Ant TH, Herd C, Louis F, Failloux AB, Sinkins SP. Wolbachia strain wAlbA blocks Zika virus transmission in Aedes aegypti. Med Vet Entomol. 2019:34:116-9.

33. Dutra HLC, Rocha MN, Dias FBS, Mansur SB, Caragata EP, Moreira LA. Wolbachia blocks currently circulating Zika virus isolates in Brazillian Aedes aegypti mosquitoes. Cell Host Microbe. 2016;19:771-4.

34. Bian G, Joshi D, Dong Y, Lu P, Zhou G, Pan X. Wolbachia invades Anopheles stephensi populations and induces refractoriness to Plasmodium infection. Science. 2013;340:748-51.

35. Kambris Z, Cook PE, Phuc HK, Sinkins SP. Immune activation by lifeshortening Wolbachia and reduced filarial competence in mosquitoes. Science. 2009:326:134-6.

36. Hughes GL, Koga R, Xue P, Fukatsu T, Rasgon JL. Wolbachia infections are virulent and inhibit the human malaria parasite Plasmodium falciparum in Anopheles gambiae. PLoS Pathog. 2011;7:e1002043.

37. Kambris Z, Blagborough AM, Pinto SB, Blagrove MSC, Godfray HCJ, Sinden RE, et al. Wolbachia stimulates immune gene expression and inhibits Plasmodium development in Anopheles gambiae. PLoS Pathog. 2010;6:e1001143.

38. Baldini F, Segata N, Pompon J, Marcenac P, Robert SW, Dabiré RK, et al. Evidence of natural Wolbachia infections in field populations of Anopheles gambiae. Nat Commun. 2014;5:3985.

39. Shaw WR, Marcenac P, Childs LM, Buckee CO, Baldini F, Sawadogo SP, et al. Wolbachia infections in natural Anopheles populations affect egg laying and negatively correlate with Plasmodium development. Nat Commun. 2016;7:11772

40. Gomes FM, Hixson BL, Tyner MDW, Ramirez JL, Canepa GE, Silva EALT, et al. Effect of naturally occurring Wolbachia in Anopheles gambiae s.l. mosquitoes from Mali on Plasmodium falciparum malaria transmission. Proc Natl Acad Sci USA. 2017;114:12566-71.

41. Nazni WA, Hoffmann AA, NoorAfizah A, Cheong YL, Mancini MV, Golding $\mathrm{N}$, et al. Establishment of Wolbachia strain wAlbB in Malaysian populations of Aedes aegypti for dengue control. Curr Biol. 2019;29:4241-8.

42. Baldini F, Rouge J, Kreppel K, Mkandawile G, Mapua SA, Sikulu-Lord M, et al. First report of natural Wolbachia infection in the malaria mosquito Anopheles arabiensis in Tanzania. Parasit Vector. 2018;11:635.

43. Jeffries CL, Lawrence GG, Golovko G, Kristan M, Orsborne J, Spence K, et al. Novel Wolbachia strains in Anopheles malaria vectors from subSaharan Africa. Wellcome Open Res. 2018:3:113.

44. Niang EHA, Bassene H, Makoundou P, Fenollar F, Weill M, Mediannikov O. First report of natural Wolbachia infection in wild Anopheles funestus population in Senegal. Malar J. 2018;17:408.

45. Ayala D, Akone-Ella O, Rahola N, Kengne P, Ngangue MF, Mezeme F, et al. Natural Wolbachia infections are common in the major malaria vectors in Central Africa. Evol Appl. 2019;12:1583-94.

46. Sawasdichai S, Chaumeau V, Dah T, Kulabkeeree T, Kajeechiwa L, Phanaphaduntham $\mathrm{M}$, et al. Natural Wolbachia infections in malaria vectors in Kayin state, Myanmar. Wellcome Open Res. 2019;4:11.

47. Carvajal TM, Hashimoto K, Harnandika RK, Amalin DM, Watanabe K. Detection of Wolbachia in field-collected Aedes aegypti mosquitoes in metropolitan Manila, Philippines. Parasit Vectors. 2019;12:361.

48. Thongsripong P, Chandler JA, Green AB, Kittayapong P, Wilcox BA, Kapan $\mathrm{DD}$, et al. Mosquito vector-associated microbiota: metabarcoding bacteria and eukaryotic symbionts across habitat types in Thailand endemic for dengue and other arthropod-borne diseases. Ecol Evol. 2017;8:1352-68.

49. Teo CHJ, Lim PKC, Voon K, Mak JW. Detection of dengue viruses and Wolbachia in Aedes aegypti and Aedes albopictus larvae from four urban local localities in Kuala Lumpur, Malaysia. Trop Biomed. 2017;34:583-97.

50. Balaji S, Jayachandran S, Prabagaran SR. Evidence for the natural occurrence of Wolbachia in Aedes aegypti mosquitoes. FEMS Microbiol Lett. 2019;366:fnz055.
51. Coon KL, Brown MR, Strand MR. Mosquitoes host communities of bacteria that are essential for development but vary greatly between local habitats. Mol Ecol. 2016;25:5806-26.

52. Kulkarni A, Yu W, Jiang J, Sanchez C, Karna AK, Martinez KJL, et al. Wolbachia pipientis occurs in Aedes aegypti populations in New Mexico and Florida, USA. Ecol Evol. 2019;9:6148-56.

53. Hegde S, Khanipov K, Albayrak L, Golovko G, Pimenova M, Saldaña MA, et al. Microbiome interaction networks and community structure from laboratory-reared and field-collected Aedes aegypti, Aedes albopictus, and Culex quinquefasciatus mosquito vectors. Front Microbiol. 2018;9:2160.

54. Bennett KL, Gómez-Martínez C, Chin Y, Saltonstall K, McMillan WO, Rovira $\mathrm{JR}$, et al. Dynamics and diversity of bacteria associated with the disease vectors Aedes aegypti and Aedes albopictus. Sci Rep. 2019;9:12160.

55. Marcon HS, Coscrato VE, Selivon D, Perondini AL, Marino CL. Variations in the sensitivity of different primers for detecting Wolbachia in Anastrepha (Diptera: Tephritidae). Braz J Microbiol. 2011;42:778-85.

56. Reid J. Anopheline mosquitoes of Malaya and Borneo. England: Staples Printer Limited; 1968.

57. Sallum MAM, Peyton EL, Harrison BA, Wilkerson RC. Revision of the Leucosphyrus group of Anopheles (Cellia) (Diptera: Culicidae). Rev Bras Entomol. 2005:49(Suppl. 1):1-152.

58. Jeffery J, Rohela M, Muslimin M, Abdul Aziz S, Jamaiah I, Kumar S, et al. Illustrated keys: some mosquitoes of Peninsula Malaysia. Kuala Lumpur: University of Malaya Press; 2012.

59. Vythilingam I, Lim YAL, Venugopalan B, Ngui R, Leong CS, Wong ML, et al. Plasmodium knowlesi malaria an emerging public health problem in Hulu Selangor, Selangor Malaysia (2009-2013): epidemiologic and entomologic analysis. Parasit Vectors. 2014;7:436.

60. Lee KS, Divis PC, Zakaria SK, Matusop A, Julin RA, Conway DJ, et al. Plasmodium knowlesi: reservoir hosts and tracking the emergence in humans and macaques. PloS Pathog. 2011;7:e1002015.

61. Zhou W, Rousset F, O'Neil S. Phylogeny and PCR-based classification of Wolbachia strains using wsp gene sequences. Proc Biol Sci. 1998;265:509-15

62. Werren $\mathrm{JH}$, Windsor DM. Wolbachia infection frequencies in insects: evidence of a global equilibrium? Proc Biol Sci. 2000;267:1277-85.

63. Kumar S, Stecher G, Tamura K. MEGA7: molecular evolutionary genetics analysis Version 70 for bigger datasets. Mol Biol Evol. 2016;33:1870-4.

64. Tan CH, Vythilingam I, Matusop A, Chan ST, Singh B. Bionomics of Anopheles latens in Kapit, Sarawak, Malaysian Borneo in relation to the transmission of zoonotic simian malaria parasite Plasmodium knowlesi. Malar J. 2008;7:52-9.

65. Wong ML, Chua TH, Leong CS, Khaw LT, Fornace K, Wan Yusoff WS, et al. Seasonal and spatial dynamics of the primary vector of Plasmodium knowlesi within a major transmission focus in Sabah, Malaysia. PloS Negl Trop Dis. 2015;9:e0004135.

66. Hii J, Kan S, Pereira M, Parmar S, Campos R, Chan M. Bancroftian filariasis and malaria in island and hinterland populations in Sabah, Malaysia. Trop Geogr Med. 1985;37:93-101.

67. Amerasinghe PH, Amerasinghe FP, Konradsen F, Fonseka KT, Wirtz RA. Malaria vectors in a traditional dry zone village in Sri Lanka. Am J Trop Med Hyg. 1999;60:421-9.

68. Feng X, Zhang S, Huang F, Zhang L, Feng J, Xia Z, et al. Biology, bionomics and molecular biology of Anopheles sinensis Wiedemann 1828 (Diptera: Culicidae), main malaria vector in China. Front Microbiol. 2017;8:1473.

69. Ramsdale CD. Internal taxonomy of the Hyrcanus group of Anopheles (Diptera, Culicidae) and its bearing on the incrimination of vectors of malaria in the west of the Palaearctic region. J Eur Mosq Control Assoc. 2001:10:1-8.

70. Sinha A, Li Z, Sun L, Carlow CKS. Complete genome sequence of the Wolbachia wAlbB endosymbiont of Aedes albopictus. Genome Biol Evol. 2019;11:706-20.

71. Chrostek E, Gerth M. Is Anopheles gambiae a natural host of Wolbachia. mBio. 2019;10:e00784.

72. Ross PA, Callahan AG, Yang Q, Jasper M, Arif M, Afizah AN, et al. An elusive endosymbiont: does Wolbachia occur naturally in Aedes aegypti? Ecol Evol. 2020;10:1581-91.

73. Zélé F, Nicot A, Berthomieu A, Weill M, Duron O, Rivero A. Wolbachia increases susceptibility to Plasmodium infection in a natural system. Proc Biol Sci. 2014;281:20132837. 
74. Osei-Poku J, Han C, Mbogo CM, Jiggins FM. Identification of Wolbachia strains in mosquito disease vectors. PloS ONE. 2012;7:e49922.

75. Weinert LA, Araujo-Jnr EV, Ahmed MZ, Welch JJ. The incidence of bacterial endosymbionts in terrestrial arthropods. Proc Biol Sci. 2015;282:20150249.

76. Hughes GL, Dodson BL, Johnson RM, Murdock CC, Tsujimoto H, Suzuki Y, et al. Native microbiome impedes vertical transmission of Wolbachia in Anopheles mosquitoes. Proc Natl Acad Sci USA. 2014;111:12498.
77. Rossi P, Ricci I, Cappelli A, Damiani C, Ulissi U, Mancini MV, et al. Mutual exclusion of Asaia and Wolbachia in the reproductive organs of mosquito vectors. Parasit Vectors. 2015;8:278.

\section{Publisher's Note}

Springer Nature remains neutral with regard to jurisdictional claims in published maps and institutional affiliations.
Ready to submit your research? Choose BMC and benefit from:

- fast, convenient online submission

- thorough peer review by experienced researchers in your field

- rapid publication on acceptance

- support for research data, including large and complex data types

- gold Open Access which fosters wider collaboration and increased citations

- maximum visibility for your research: over 100M website views per year

At BMC, research is always in progress.

Learn more biomedcentral.com/submissions 